\title{
The Neutrophil-to-Lymphocyte Ratio (NLR) Predicts the Prognosis of Unresectable Intermediate and Advanced Hepatocellular Carcinoma Treated with Apatinib
}

Huaqi Wang, '* Zhiwei Wang, (D) I,* Zhenyu Hou,' Xuejiao Yang,' Keyun Zhu,' Manqing Cao, ${ }^{2}$ Xiaolin Zhu, ' Huikai Li, ' Ti Zhang ',3

'Department of Hepatobiliary Surgery, Tianjin Medical University Cancer Institute and Hospital; Liver Cancer Center, Tianjin Medical University Cancer Institute and Hospital; National Clinical Research Center for Cancer; Key Laboratory of Cancer Prevention and Therapy, Tianjin; Tianjin's Clinical Research Center for Cancer, Tianjin, 300060, People's Republic of China; ${ }^{2}$ Department of Breast Surgery, Tianjin Medical University Cancer Institute \& Hospital, National Clinical Research Center for Cancer, Key Laboratory of Cancer Prevention and Therapy, Tianjin, People's Republic of China; ${ }^{3}$ Department of Hepatic Surgery, Fudan University Shanghai Cancer Center, Shanghai Medical College, Fudan University, Shanghai, 200032, People's Republic of China

*These authors contributed equally to this work

Correspondence: Ti Zhang

Department of Hepatic Surgery, Fudan University Shanghai Cancer Center, Shanghai Medical College, Fudan University, Shanghai, 200032, People's

Republic of China

$\mathrm{Tel} / \mathrm{Fax}+86-2 \mathrm{I}-6417-5590$

Email zhangti@shca.org.cn

Huikai Li

Department of Hepatobiliary Surgery, Tianjin Medical University Cancer Institute and Hospital, Key Laboratory of Cancer Prevention and Therapy, National Clinical Research Center for Cancer, 24 Bin Shui Road, Hexi District, Tianjin, 300060, People's Republic of China $\mathrm{Tel} / \mathrm{Fax}+86-22-2335-9984$

Email tjchlhk@I26.com
Purpose: Patients with hepatocellular carcinoma (HCC) who might benefit most from antiangiogenesis therapy remain unknown. In recent years, neutrophil-to-lymphocyte ratio (NLR), an indicator of inflammatory response, has received particular attention in HCC. Herein, we explored the prognostic value of pre-treatment NLR in individuals with unresectable intermediate and advanced hepatocellular carcinoma treated with apatinib, a secondline angiogenesis inhibitor. The findings of this study would assist in precision medicine and provide clinical decision support.

Patients and Methods: This is a retrospective study in which 171 HCC patients attending Tianjin Medical University Cancer Institute and Hospital and treated with apatinib between January 2016 and July 2018 were enrolled. The prognosis of the patients based on NLR signatures was then analyzed.

Results: Patients with a low pre-treatment NLR (NLR < 2.49) presented a significantly longer overall survival (OS) $(\mathrm{P}<0.001)$ and progression-free survival (PFS) $(\mathrm{P}=0.043)$. Furthermore, a low pre-treatment NLR level could be used to predict a longer OS in patients with non-macrovascular invasion $(\mathrm{P}<0.001)$. Independent of serum alpha-fetoprotein (AFP) levels, a low NLR level in this cohort of patients is associated with a longer OS.

Conclusion: Pre-treatment NLR predicts the prognosis of patients with unresectable intermediate and advanced HCC treated with apatinib.

Keywords: hepatocellular carcinoma, vascular endothelial growth factor receptor - 2, neutrophil to lymphocyte ratio, tyrosine protein kinases inhibitor

\section{Introduction}

Hepatocellular carcinoma (HCC) is the sixth most prevalent malignant tumor and the third leading cause of cancer-related deaths. ${ }^{1}$ Approximately $40 \%$ of HCC patients are diagnosed at advanced stages and the majority of them have limited access to radical treatments. ${ }^{2}$ Sorafenib and lenvatinib, two tyrosine protein kinase inhibitors (TKI), are the major targeted therapies against $\mathrm{HCC}^{3-5}$ Indeed, compared to placebo, sorafenib was demonstrated to be effective by prolonging the median OS by 3 months, and lenvatinib presented a non-inferiority OS to that of sorafenib. ${ }^{5,6}$ Apatinib is a novel highly selective small-molecule tyrosine kinase inhibitor of vascular endothelial growth factor receptor (VEGFR)-2, with a strong antitumor activity against numerous cancers, including gastric cancer, breast cancer, ovarian cancer and liver cancer. ${ }^{7-11}$ 
A Phase III clinical trial has demonstrated that compared to placebo, apatinib markedly improves the objective response rate (ORR) and is thus recommended as a second-line treatment for advanced HCC. ${ }^{12}$ Apatinib also substantially prolongs the OS of patients with sorafenib-refractory advanced HCC. ${ }^{13}$ In addition, apatinib may also present its vital and outstanding characteristics in optimization and regulation in tumor immune microenvironment, which exerts synergistic anti-cancer effects in combination with immunotherapy. ${ }^{14,15}$ However, the lack of the predictive biomarkers for antiangiogenesis therapy has been a challenge over the past few years. So far, there are no validated biomarkers for predicting the efficacy of apatinib treatment for HCC.

Literature illustrates that inflammation participates in the development and progression of HCC. ${ }^{16,17}$ Furthermore, preclinical findings suggest that combination therapy of targeted therapy and immunotherapy is superior to either module alone, which indicates the synergistic antitumor effects of Programmed Cell Death Protein 1 (PD-1) blockade with apatinib in HCC. ${ }^{18}$ Neutrophil-to-lymphocyte ratio (NLR), one of the inflammatory markers, has recently been studied extensively. ${ }^{19}$ More and more evidence indicates that NLR is considered as a prognostic predictor for HCC after curative resection, liver transplantation and sorafenib therapy. ${ }^{20-22}$ Herein, our finding suggests that NLR may be an accurate prognostic marker for OS and PFS of unresectable intermediate and advanced HCC patients on apatinib treatment.

\section{Patients and Methods}

A total of 171 patients with unresectable intermediate and advanced HCC who received apatinib treatment between January 2016 and July 2018 at Tianjin Medical University Cancer Institute and Hospital were enrolled for this study. The median follow-up period was 29.8 months. Clinical and biodata were extracted from medical records. Inclusion criteria for the study were as follows: (a) 18 years or older with unresectable Barcelona Clinical Liver Cancer (BCLC) stage B or C HCC; (b) previously refractory or intolerant to at least one line of systemic therapy; (c) adequate liver function: Child-Pugh A or B (bilirubin $\leq 3 \mathrm{mg} / \mathrm{dl}$, ALT and $\mathrm{AST} \leq 5$ times the upper limit of normal level) and (d) complete and available data on whole-blood count. Those (a) currently or previously on splenectomy treatment; (b) with refractory ascites; (c) infectious or hematologic disorders and (d) other autoimmune diseases were all excluded from the study. NLR is defined as the absolute neutrophil-to-lymphocyte ratio. Baseline
NLR was performed on blood collected within 14 days of cancer diagnosis before initiation of apatinib therapy.

\section{Treatment and Follow-Up}

All patients received a daily dose of $250 \mathrm{mg}$ or $500 \mathrm{mg}$ of apatinib and were followed up routinely until the cut-off date 30 September 2020. The routine examinations were as follows: blood routines, AFP, liver function, abdomen ultrasonography every three months, and contrast CT or magnetic resonance imaging (MRI) every six months. Overall survival (OS) was defined as the interval from the commencement of apatinib to death for any reason or the last date of follow-up. Progression-free survival (PFS) is defined as the interval from the commencement of apatinib to disease progression or death from any cause.

\section{Statistical Analysis}

All statistical analysis was assessed using SPSS 25.0 (IBM Corporation, Armonk, NY, USA) and Prism software (GraphPad Prism Software, La Jolla, CA, USA). Quantitative values were analyzed using Student's $t$-test. Categorical variables were analyzed using the Chi-squared tests. The accuracy of NLR in predicting the OS and PFS of HCC patients was evaluated using Kaplan-Meier method. The Cox proportional hazards model was performed for univariate and multivariate analyses. $p<0.05$ was considered statistically significant.

\section{Results}

\section{Baseline Characteristics of the HCC Patients Treated with Apatinib}

Of the 171 patients, 149 (87.1\%) were males, whereas 22 $(12.9 \%)$ were females. The mean and median NLR prior initiation of treatment were 2.64 and 2.21 , respectively. Based on receiver operating characteristic curves (ROC), the optimal cutoff value of NLR for better prognosis of HCC patients was 2.49. The patients were then categorized into NLR-high (NLR $\geq 2.49, \mathrm{n}=70)$ and NLR-low (NLR $<$ $2.49, \mathrm{n}=101$ ) group based on median NLR. The clinical characteristics of patients in the two groups are shown in Table 1. Overall, $32(45.7 \%)$ and $35(50 \%)$ patients in the NLR-high group presented with high AFP level (AFP $>400$ $\mathrm{ng} / \mathrm{mL}$ ) and macrovascular invasion, respectively. In the NLR-low group, high AFP level and macrovascular invasion were observed in $52(51.5 \%)$ and $48(47.5 \%)$ patients, respectively. The pre-treatment NLR was closely associated with Eastern Cooperative Oncology Group performance 
Table I Baseline Characteristics of the HCC Patients

\begin{tabular}{|c|c|c|c|}
\hline Variables & $N L R \geq 2.49(n=70)$ & NLR $<2.49(n=101)$ & P-value \\
\hline Age, $n(\%)$, years & & & 0.206 \\
\hline$<60$ & $37(52.9)$ & $64(63.4)$ & \\
\hline$\geq 60$ & $33(47.1)$ & $37(36.6)$ & \\
\hline Gender, n(\%) & & & 1.000 \\
\hline Male & $6 I(87.1)$ & $88(87.1)$ & \\
\hline Female & $9(12.9)$ & $13(12.9)$ & \\
\hline AFP, $n(\%), n g / m L$ & & & 0.534 \\
\hline$\leq 400$ & $38(54.3)$ & $49(48.5)$ & \\
\hline$>400$ & $32(45.7)$ & $52(51.5)$ & \\
\hline ECOG PS, n(\%) & & & 0.008 \\
\hline 0 & $25(35.7)$ & $58(57.4)$ & \\
\hline I & $45(64.3)$ & $43(42.6)$ & \\
\hline Child-Pugh, n(\%) & & & 0.006 \\
\hline A & $46(65.7)$ & $85(84.2)$ & \\
\hline B & $24(34.3)$ & $16(15.8)$ & \\
\hline Tumor size, cm, n(\%) & & & 0.873 \\
\hline$\leq 10$ & $50(71.4)$ & $71(70.3)$ & \\
\hline$>10$ & $20(28.6)$ & $30(29.7)$ & \\
\hline Tumor number, n(\%) & & & 0.136 \\
\hline$\leq 3$ & $30(42.9)$ & $55(54.5)$ & \\
\hline$>3$ & $40(57.1)$ & $46(45.5)$ & \\
\hline BCLC stage, $n(\%)$ & & & 0.846 \\
\hline B & $14(20)$ & $19(18.8)$ & \\
\hline $\mathrm{C}$ & $56(80)$ & $82(81.2)$ & \\
\hline \multicolumn{4}{|l|}{ Type of Hepatitis, n(\%) } \\
\hline None & $14(20)$ & $15(14.9)$ & 0.412 \\
\hline HBV & $50(71.4)$ & $84(83.2)$ & 0.089 \\
\hline $\mathrm{HCV}$ & $4(5.7)$ & $\mathrm{I}(\mathrm{I} .0)$ & 0.160 \\
\hline $\mathrm{HBV}+\mathrm{HCV}$ & $2(2.9)$ & $\mathrm{I}(\mathrm{I} .0)$ & 0.568 \\
\hline Macrovascular invasion, $\mathrm{n}(\%)$ & $35(50.0)$ & $48(47.5)$ & 0.758 \\
\hline Extrahepatic spread, n(\%) & $37(52.9)$ & $56(55.4)$ & 0.757 \\
\hline Bone metastasis, $n(\%)$ & $9(12.9)$ & $\mathrm{II}(10.9)$ & 0.810 \\
\hline Lung metastasis, n(\%) & $10(14.3)$ & $6(5.9)$ & 0.107 \\
\hline Lymph node metastasis, $\mathrm{n}(\%)$ & $27(38.6)$ & $38(37.6)$ & 0.873 \\
\hline \multicolumn{4}{|l|}{ Prior treatment, $n(\%)$} \\
\hline Surgery & $16(22.9)$ & $40(39.6)$ & 0.031 \\
\hline RFA & $6(8.6)$ & $15(14.9)$ & 0.246 \\
\hline TACE or TAE & $58(82.9)$ & $75(74.3)$ & 0.197 \\
\hline Radiotherapy & $4(5.7)$ & $3(3.0)$ & 0.446 \\
\hline $\mathrm{HGB}, \mathrm{g} / \mathrm{L}^{*}$ & $137 \pm 22$ & $146 \pm 19$ & 0.003 \\
\hline WBC, $10^{\wedge} 9 / L^{*}$ & $6.09 \pm 1.74$ & $5.32 \pm 1.54$ & 0.003 \\
\hline PLT, I0^9/L $\dagger$ & $174(107,211)$ & $163(100,210)$ & 0.538 \\
\hline ALT, U/L† & $46(23,58)$ & $4 I(24,48)$ & 0.594 \\
\hline
\end{tabular}

(Continued) 
Table I (Continued).

\begin{tabular}{|l|l|l|l|}
\hline Variables & NLR $\geq \mathbf{2 . 4 9}(\mathbf{n = 7 0})$ & $\mathbf{N L R < 2 . 4 9}(\mathbf{n = 1 0 1})$ & P-value \\
\hline AST, U/L† & $61(34,75)$ & $57(30,77)$ & 0.291 \\
\hline TBil, $\mu \mathrm{mol} / \mathrm{L} \dagger$ & $24.6(15.3,32.3)$ & $22.5(15.3,28.8)$ & 0.500 \\
\hline ALB, g/L* & $37.3 \pm 5.0$ & $39.9 \pm 5.8$ & 0.003 \\
\hline GGT, U/L $\dagger$ & $236(98,321)$ & $169(66,224)$ & 0.017 \\
\hline
\end{tabular}

Notes: *Student's t-test. †Mann-Whitney U-test.

Abbreviations: NLR, neutrophil to lymphocyte ratio; ECOG PS, Eastern Cooperative Oncology Group performance status score; BCLC, Barcelona Clinic Liver Cancer; HBV, hepatitis B virus; HCV, hepatitis C virus; AFP, alpha-fetoprotein; TACE, transcatheter arterial chemoembolization; TAE, transcatheter arterial embolization; RF, radiofrequency ablation; HGB, hemoglobin; WBC, white blood cell count; PLT, platelet count; ALT, glutamic-pyruvic transaminase; AST, aspartate aminotransferase; ALB, albumin; TBil, total bilirubin; GGT, gamma-glutamyl transpeptidase.

status score (ECOG PS), Child-Pugh score, surgery, hemoglobin (HGB) levels, white blood cell (WBC) count, albumin (ALB) level and serum gamma-glutamyl transpeptidase (GGT) level $(\mathrm{P}<0.05)$. No obvious correlations with age, gender, serum AFP levels, BCLC stage, hepatitis virus, platelet count (PLT), glutamic-pyruvic transaminase (ALT), aspartate aminotransferase (AST) or total bilirubin (TBil) were observed $(\mathrm{P}>0.05)$.

\section{Factors Associated with Prognosis of HCC Patients on Apatinib Treatment}

To identify the influencing factors for prognosis after the administration of apatinib, univariable and multivariable analyses were performed. Univariate analysis revealed that ALB level, GGT level, total bilirubin (TBil), Child-Pugh score, tumor size, tumor number, macrovascular invasion and surgery substantially influenced the OS, whereas ALB level, ALT level and radiofrequency ablation (RFA) significantly influenced the PFS in this cohort (Table 2). Multivariate analysis further revealed that total bilirubin (TBil), ALB level, and RFA were the most significant factors influencing the OS, whereas ALT level and RFA were associated with the PFS in this cohort. These findings demonstrate that pre-treatment NLR is an independent prognostic factor for predicting both OS and PFS of $\mathrm{HCC}$ patients treated with apatinib.

\section{Overall and Progression-Free Survival According to NLR}

To evaluate the value of pre-treatment NLR in predicting OS and PFS, Kaplan-Meier method was performed. KaplanMeier survival analysis revealed that the median OS of patients in the low NLR group was significantly higher than that of those in the high NLR group (17.15 months vs
10.91 months; $\mathrm{p}<0.001$ ) (Figure 1A). Comparable findings were observed for median PFS (7.92 months vs 6.46 months; $p=0.043$ ) (Figure 1B). In general, our research demonstrated that a high pre-treatment NLR level in HCC patients treated with apatinib implied poor OS and PFS.

\section{Prognostic Significance of NLR Within AFP, Macrovascular Invasion}

Considering that two variables (AFP and macrovascular invasion) were associated with overall survival, subgroup analysis was performed. Patients were classified into four groups based on mean serum AFP levels and NLR values. The results are shown in Figure 2. We found NLR $\geq 2.49$ tended to be a predictor of poor OS regardless of serum AFP levels (Figure 2A). In addition, NLR could be used as a predictor of OS for patients with non-macrovascular invasion group $(\mathrm{p}<$ 0.001). However, NLR was not a prognosis predictor of OS in $\mathrm{HCC}$ patients with macrovascular infiltration (Figure 2B).

\section{Safety}

Drug-related deaths did not occur in this study, and also no grade 5 drug-related adverse events occurred in all patients. The adverse events that occurred in $\geq 10 \%$ of patients are shown in Table 3. There were no new drug-related adverse events, and this was consistent with previously reported adverse events. ${ }^{12,23}$ The most common AEs were hypertension (43.3\%), hand-foot syndrome (39.2\%), fatigue (34.5\%), hepatic insufficiency (29.8\%), hematological toxicity (29.8\%), anorexia (25.1\%) and proteinuria (24.6\%). Any hepatic insufficiency included elevated aspartate or alanine aminotransferase levels, hyperbilirubinemia. Grade 3-4 AE included proteinuria (12.9\%), hematological toxicity $(11.1 \%)$, hand-foot syndrome $(5.8 \%)$ and hypertension 


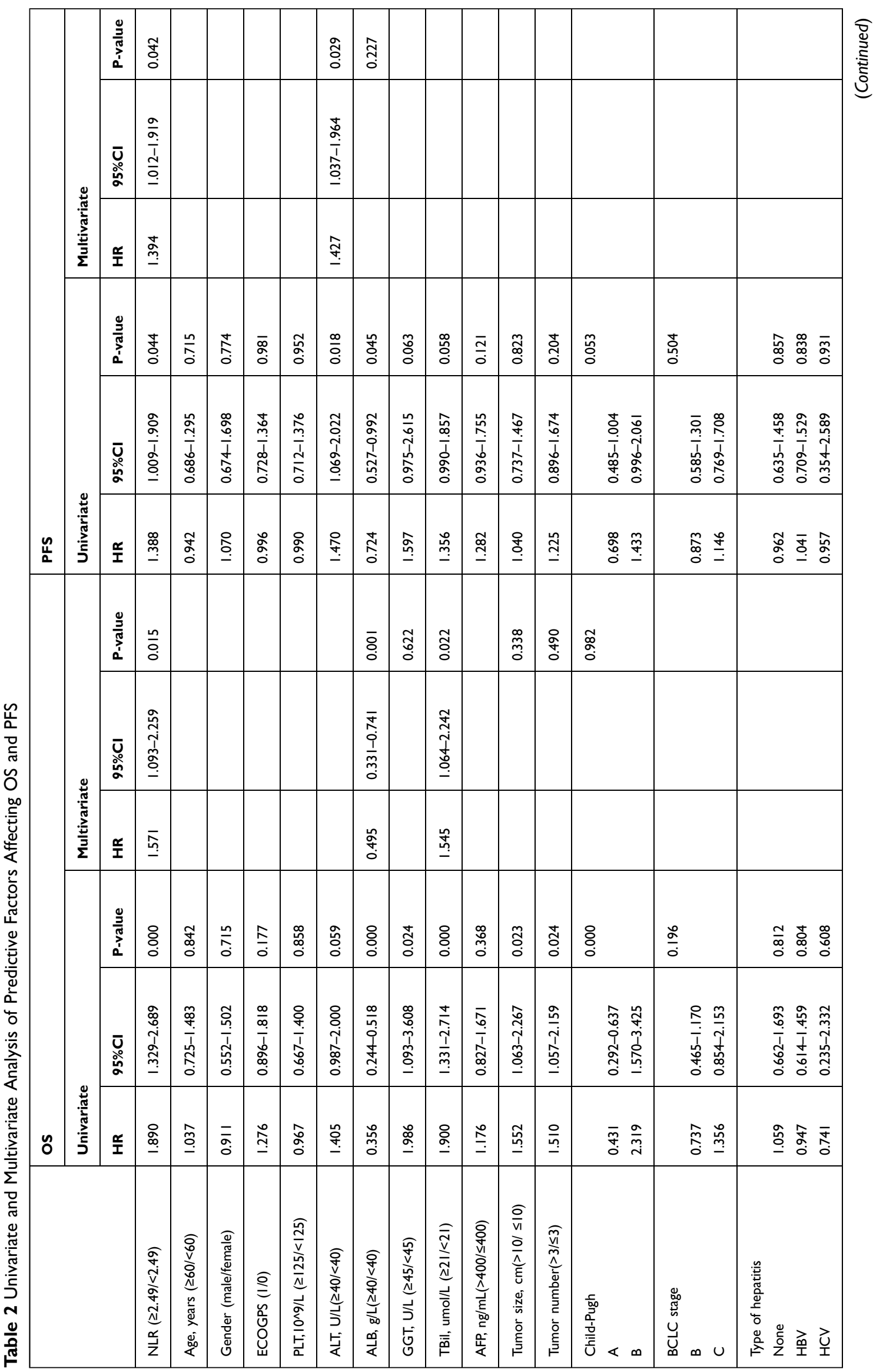




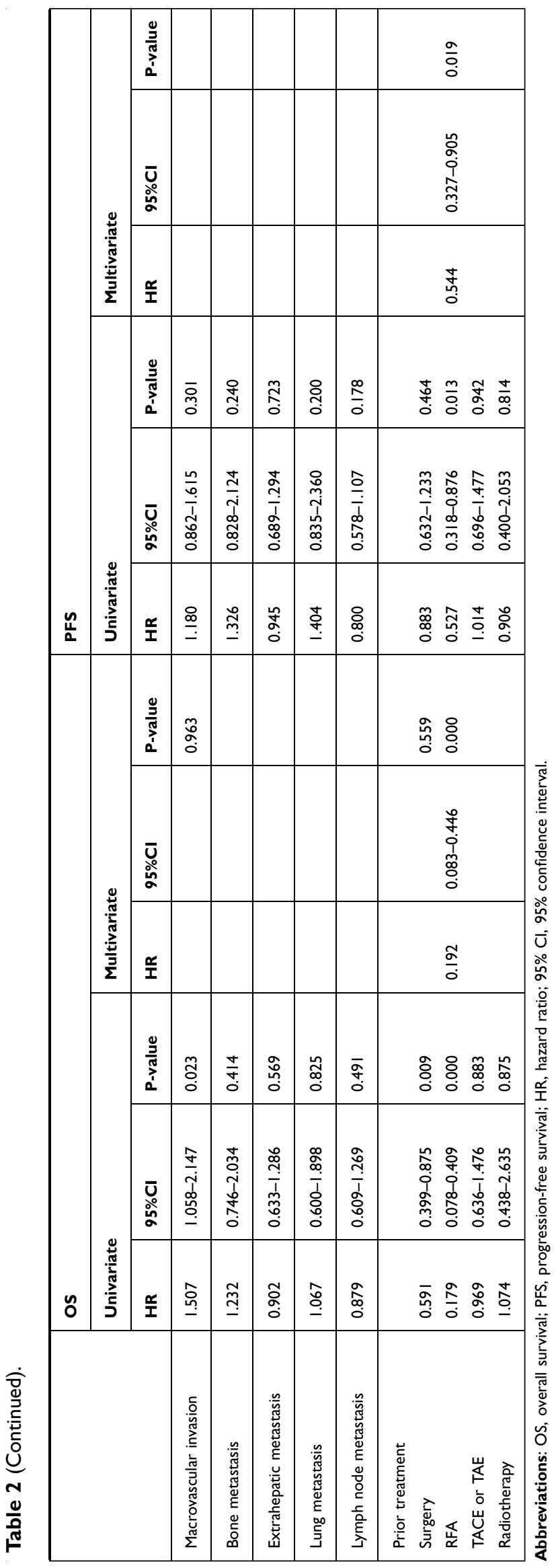

(1.2\%). Any drug-related AEs could be recovered by dose reduction or discontinuation.

\section{Discussion}

Although anti-angiogenic therapy has achieved remarkable efficacy in advanced HCC treatment, there is no accurate biomarker for assessing treatment response to targeted therapy. ${ }^{5,24-26}$ In numerous studies, NLR has the potential to serve as a predictor for various cancers, including lung cancer, gastric cancer, breast cancer, esophageal carcinoma, renal carcinoma and biliary tract cancer. ${ }^{27-33}$ In the present study, we evaluated the prognostic utility of NLR for unresectable HCC on apatinib treatment. Patients with unresectable intermediate and advanced $\mathrm{HCC}$ on apatinib treatment were divided into two groups based on the optimal cutoff value of NLR. We found that high pretreatment NLR was associated with poorer prognosis with relative to low NLR. This may indicate that pre-treatment NLR grading could play an important role in predicting the survival outcomes. Given that NLR is an easily accessible index in the clinical settings, it possesses potential practical applications for assisting clinicians in distinguishing HCC patients presenting poor survival with apatinib treatment.

Since NLR in early and intermediate stage HCC has been discussed, our research provides an important supplement in patients with unresectable intermediate and advanced HCC. ${ }^{34}$ We suggest NLR as a convenient indicator to select patients suitable for systemic therapy. In particular, when subgroup stratification was performed based on AFP and macrovascular invasion, the survival differences between subgroups underscored the importance of NLR grading. Regardless of serum AFP levels, NLR $<2.49$ may be served as a predictor of a longer OS. In the subgroup analysis of patients with nonmacrovascular invasion, patients with low NLR presented a better OS than those with high NLR.

Recently, novel immunotherapy has been a breakthrough in the treatment of HCC. HCC is one of inflammation-related cancers and may evade the immune system by targeting immune checkpoints, such as cytotoxic programmed death receptor 1 (PD-1) or its ligand (PD-L1), T-lymphocyte associated protein 4 (CTLA-4), among many others. ${ }^{35}$ In the process of tumorigenesis and development, neutrophils release factors to stimulate cancer cell proliferation and are recognized as negative regulators of anti-tumor immunotherapy. ${ }^{36}$ Lymphocytes act as warriors against tumor progression, and as such, 
A

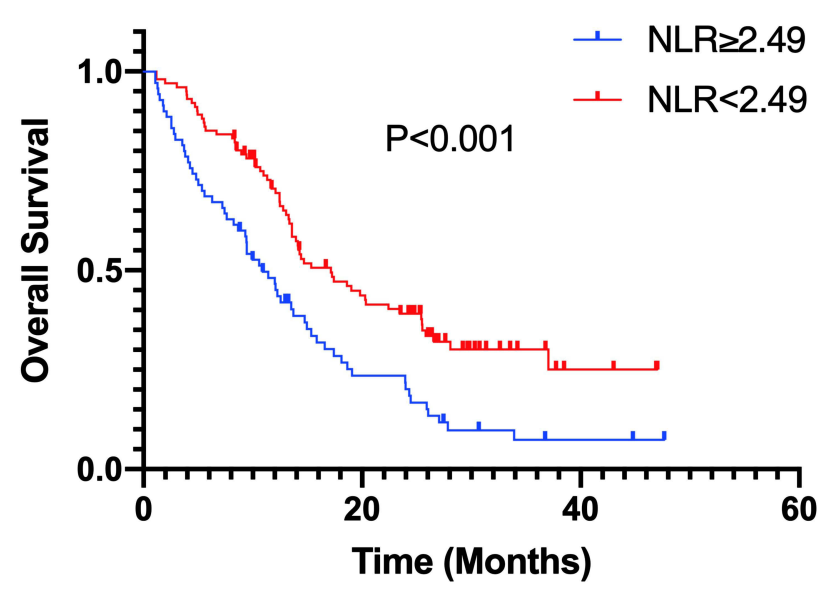

B

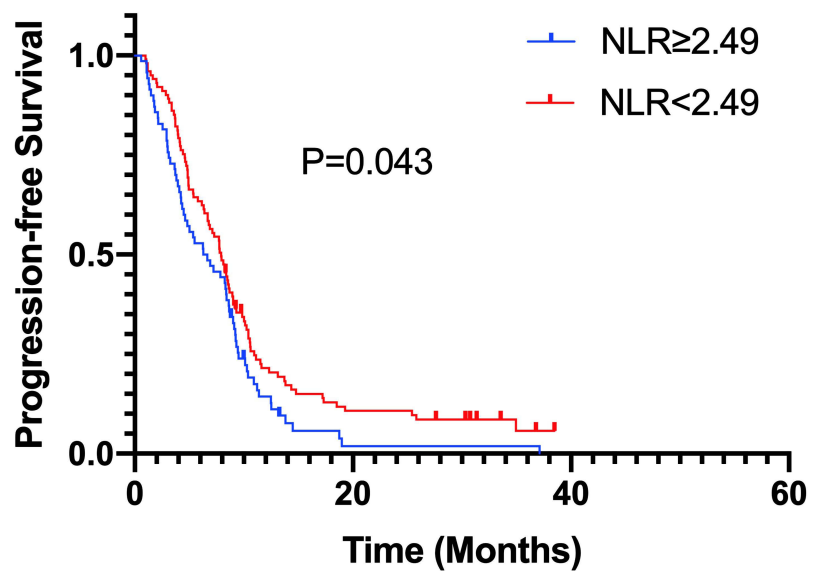

Figure I Kaplan-Meier survival analysis of OS and PFS. High NLR $\geq 2.49$ was associated with a shorter OS (A) and PFS (B).

A

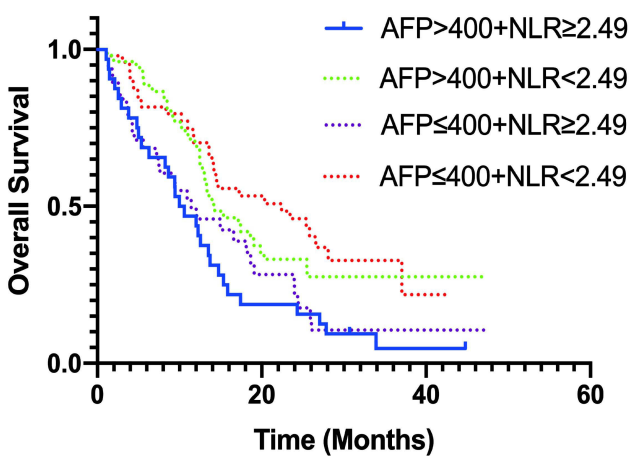

B

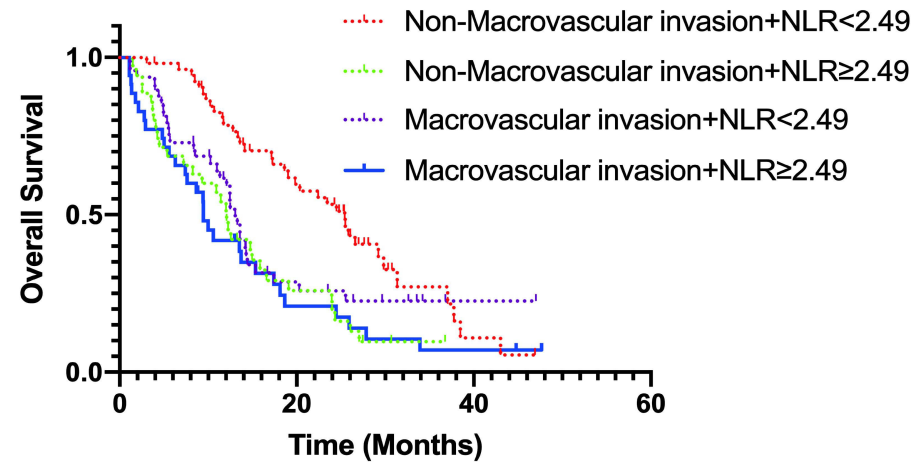

Figure 2 Subgroup analysis of OS. (A) Subgroup with AFP $\leq 400 \mathrm{ng} / \mathrm{mL}$, the median OS (NLR-low vs NLR-high, $22.4 \mathrm{I}$ months vs II.43 months, $\mathrm{P}=0.008$ ) was significantly better in the NLR-low group than in the NLR-high group; subgroup with AFP > 400ng/mL, the median OS (NLR-low vs NLR-high, 14.29 months vs I0.29 months, $\mathrm{P}=0.007$ ) was also better in the NLR-low group than in the NLR-high group. (B) Subgroup with non-macrovascular invasion, the median OS (NLR-low vs NLR-high, 25.40 months vs 12.09 months, $\mathrm{p}<0.00 \mathrm{I}$ ) was significantly better in the NLR-low group than in the NLR-high group; subgroup with macrovascular invasion, NLR cannot be used as a predictor of OS.

relative lymphocyte depletion may represent an initial state of resistance to immunotherapy. ${ }^{37}$ A high NLR level may reflect increased neutrophil response or decreased lymphocyte response, and consequently, it possibly indicates a poor immunity status, resulting in a worse prognosis. A preclinical study on advanced HCC revealed that PD-1 antibody or PD-L1 antibody were less efficacious in non-inflamed tumors characterized by a low ratio of tumor-associated lymphocytes. Once VEGFR-2 blockade was administered in combination with PD-1 or PD-L1 antibody, it reversed the deficiency in lymphocytes infiltration both in serum and in tumor tissues. ${ }^{38}$ Interestingly, dual VEGFR and PD-L1 blockade has shown increased
OS in advanced lung cancer patients in clinical setting. ${ }^{39}$ As such, antiangiogenic drugs like apatinib are thought to reverse the immune inflammatory state and restore normal immune responses, thus facilitating the efficacy of immunotherapy. In other words, patients with high NLR may be more responsive to immunotherapy in combination with apatinib, relative to those with low NLR who respond better to apatinib monotherapy. As a potential inflammatory marker, NLR is speculated to play a vital guiding role in predicting the response to antiangiogenic drugs combined with immunotherapy. Due to the limited cases of combination therapy of apatinib and PD-1 antibody, little is known about the underlying mechanisms of synergistic 
Table 3 Adverse Events

\begin{tabular}{|l|l|l|}
\hline Adverse Events & Any Grade, n(\%) & Grade 3 or 4, n(\%) \\
\hline Hypertension & $74(43.3 \%)$ & $2(1.2 \%)$ \\
Hand-foot-syndrome & $67(39.2 \%)$ & $10(5.8 \%)$ \\
Fatigue & $59(34.5 \%)$ & $6(3.5 \%)$ \\
Hepatic insufficiency & $51(29.8 \%)$ & $9(5.3 \%)$ \\
Hematological toxicity & $51(29.8 \%)$ & $19(11.1 \%)$ \\
Anorexia & $43(25.1 \%)$ & $9(5.3 \%)$ \\
Proteinuria & $42(24.6 \%)$ & $22(12.9 \%)$ \\
Diarrhea & $39(22.8 \%)$ & $1(0.6 \%)$ \\
Vomiting & $25(14.6 \%)$ & $2(1.2 \%)$ \\
Hoarse voice & $23(13.5 \%)$ & $2(1.2 \%)$ \\
Dry mouth & $22(12.9 \%)$ & 0 \\
\hline
\end{tabular}

anti-tumor effects of antiangiogenic drugs and immunotherapy as well as the relationship between NLR and combination therapy. More cases are indeed to be collected and analyzed further, so that patients could receive optimal therapy schemes based on a given NLR. In the next coming years, the combination of apatinib plus immunotherapy may largely benefit patients with unresectable intermediate and advanced HCC, and this haematological index could guide clinicians to make the right therapeutic decisions.

The present study is retrospective and has its limitations in nature. First, selection bias cannot be ruled out. Second, it is a single-center study with a varied number of patients and the sample size is relatively small; further studies with a larger cohort of patients are needed to justify this finding. Third, the study is limited to an Asian population, it cannot fully represent the overall population.

\section{Conclusion}

Our study supports further investigation of NLR as an available and inexpensive pre-treatment marker. This work is an important supplement to the evidence that such a marker could be used to prognosticate and predict the outcomes of $\mathrm{HCC}$ patients treated with antiangiogenesis.

\section{Ethics Approval}

The protocol for this study was approved by the Medical Ethics Committee of the Tianjin Medical University Cancer Institute and Hospital (reference number bc2021004), and this retrospective study was conducted in accordance with Helsinki Declaration of 1975. Since this study was a retrospective review of the medical records and all clinical and biodata have been anonymously processed, obtaining informed consents from the individuals included was not deemed necessary and not required by the ethical review board.

\section{Acknowledgments}

We are grateful to all the patients and their relatives included for their support and understanding. We also thank XiaoYing Gu, Su Zhang, XiHao Zhang, Tao Guan, $\mathrm{Zhou} \mathrm{Fu}$ for their contribution to this work.

\section{Author Contributions}

Ti Zhang and Hui-Kai Li conceptualized and designed the study. All authors participated in the acquisition, analysis and interpretation of the data. Hua-Qi Wang and Zhi-Wei Wang drafted the manuscript. Zhen-Yu Hou, Xue-Jiao Yang, Ke-Yun Zhu, Man-Qing Cao and Xiao-Lin Zhu contributed to critical revisions of the manuscript. All authors agreed on the journal to which the article would be submitted, gave final approval for the version to be published, and agreed to be accountable for all aspects of the work.

\section{Disclosure}

This study was funded by the National Natural Science Foundation of China (No. 81672884) and the National Science and Technology Major Project of China (No. 2017ZX10203207-004-005). The authors declare no conflicts of interest.

\section{References}

1. Forner A, Reig M, Bruix J. Hepatocellular carcinoma. Lancet. 2018;391(10127):1301-1314. doi:10.1016/S0140-6736(18)30010-2

2. Ikeda M, Morizane C, Ueno M, Okusaka T, Ishii H, Furuse J. Chemotherapy for hepatocellular carcinoma: current status and future perspectives. Jpn J Clin Oncol. 2018;48(2):103-114. doi:10.1093/jjco/ hyx 180

3. Cheng AL, Kang YK, Chen Z, et al. Efficacy and safety of sorafenib in patients in the Asia-Pacific region with advanced hepatocellular carcinoma: a phase III randomised, double-blind, placebo-controlled trial. Lancet Oncol. 2009;10(1):25-34. doi:10.1016/S1470-2045(08)702857

4. Benson AB, D'Angelica MI, Abbott DE, et al. Guidelines insights: hepatobiliary cancers, version 2.2019. J Natl Compr Canc Netw. 2019;17(4):302-310. doi:10.6004/jnccn.2019.0019

5. Kudo M, Finn RS, Qin S, et al. Lenvatinib versus sorafenib in first-line treatment of patients with unresectable hepatocellular carcinoma: a randomised Phase 3 non-inferiority trial. Lancet. 2018;391 (10126):1163-1173. doi:10.1016/S0140-6736(18)30207-1

6. Meyer T. Treatment of advanced hepatocellular carcinoma: beyond sorafenib. Lancet Gastroenterol Hepatol. 2018;3(4):218-220. doi:10.1016/S2468-1253(17)30255-8

7. Yang C, Qin S. Apatinib targets both tumor and endothelial cells in hepatocellular carcinoma. Cancer Med. 2018;7(9):4570-4583. doi:10.1002/cam4.1664 
8. Li J, Qin $\mathrm{S}$, $\mathrm{Xu} \mathrm{J}$, et al. Randomized, double-blind, placebo-controlled Phase III trial of apatinib in patients with chemotherapy-refractory advanced or metastatic adenocarcinoma of the stomach or gastroesophageal junction. J Clin Oncol. 2016;34 (13):1448-1454. doi:10.1200/JCO.2015.63.5995

9. Hu X, Cao J, Hu W, et al. Multicenter Phase II study of apatinib in non-triple-negative metastatic breast cancer. BMC Cancer. 2014;14 (1):820. doi:10.1186/1471-2407-14-820

10. Miao M, Deng G, Luo S, et al. A phase II study of apatinib in patients with recurrent epithelial ovarian cancer. Gynecol Oncol. 2018;148(2):286-290. doi:10.1016/j.ygyno.2017.12.013

11. Du X, Chen D, Lin Z, et al. Efficacy of apatinib in advanced hepatocellular carcinoma with lung metastasis: a retrospective, multicenter study. J BUON. 2019;24(5):1956-1963.

12. Qin S, Li Q, Gu S, et al. Apatinib as second-line or later therapy in patients with advanced hepatocellular carcinoma (AHELP) a multicentre, double-blind, randomised, placebo-controlled, phase 3 trial. Lancet Gastroenterol Hepatol. 2021;6(7):559-568. doi:10.1016/S2468-1253(21)00109-6

13. Zhang Y, Fan W, Wang Y, Huang G, Li J. Apatinib for patients with sorafenib-refractory advanced hepatitis B virus related hepatocellular carcinoma: results of a Pilot Study. Cancer Control. 2019;26 (1):1073274819872216. doi:10.1177/1073274819872216

14. Rizzo A, Ricci AD, Brandi G. Atezolizumab in advanced hepatocellular carcinoma: good things come to those who wait. Immunotherapy. 2021;13(8):637-644. doi:10.2217/imt-2021-0026

15. Mei K, Qin S, Chen Z, Liu Y, Wang L, Zou J. Camrelizumab in combination with apatinib in second-line or above therapy for advanced primary liver cancer: cohort a report in a multicenter phase Ib/II trial. J Immunother Cancer. 2021;9(3):e002191. doi:10.1136/jitc-2020-002191

16. Ringelhan M, Pfister D, O’Connor T, Pikarsky E, Heikenwalder M. The immunology of hepatocellular carcinoma. Nat Immunol. 2018;19 (3):222-232. doi:10.1038/s41590-018-0044-z

17. Endig J, Buitrago-Molina LE, Marhenke S, et al. Dual role of the adaptive immune system in liver injury and hepatocellular carcinoma development. Cancer Cell. 2016;30(2):308-323. doi:10.1016/j. ccell.2016.06.009

18. Yang Y, Wang C, Sun H, Jiang Z, Zhang Y, Pan Z. Apatinib prevents natural killer cell dysfunction to enhance the efficacy of anti-PD-1 immunotherapy in hepatocellular carcinoma. Cancer Gene Ther. 2021;28(1-2):89-97. doi:10.1038/s41417-020-0186-7

19. Motomura T, Shirabe K, Mano Y, et al. Neutrophil-lymphocyte ratio reflects hepatocellular carcinoma recurrence after liver transplantation via inflammatory microenvironment. $J$ Hepatol. 2013;58 (1):58-64. doi:10.1016/j.jhep.2012.08.017

20. Limaye AR, Clark V, Soldevila-Pico C, et al. Neutrophil-lymphocyte ratio predicts overall and recurrence-free survival after liver transplantation for hepatocellular carcinoma. Hepatol Res. 2013;43 (7):757-764. doi:10.1111/hepr.12019

21. Gomez D, Farid S, Malik HZ, et al. Preoperative neutrophil-tolymphocyte ratio as a prognostic predictor after curative resection for hepatocellular carcinoma. World J Surg. 2008;32(8):1757-1762. doi:10.1007/s00268-008-9552-6

22. Bruix J, Cheng AL, Meinhardt G, Nakajima K, De Sanctis Y, Llovet J. Prognostic factors and predictors of sorafenib benefit in patients with hepatocellular carcinoma: analysis of two phase III studies. $J$ Hepatol. 2017;67(5):999-1008. doi:10.1016/j. jhep.2017.06.026

23. Hou Z, Zhu K, Yang X, et al. Apatinib as first-line treatment in patients with advanced hepatocellular carcinoma: a phase II clinical trial. Ann Transl Med. 2020;8(17):1047. doi:10.21037/atm-20-2990
24. Llovet JM, Ricci S, Mazzaferro V, et al. Sorafenib in advanced hepatocellular carcinoma. $N$ Engl J Med. 2008;359(4):378-390. doi:10.1056/NEJMoa0708857

25. Bruix J, Qin S, Merle P, et al. Regorafenib for patients with hepatocellular carcinoma who progressed on sorafenib treatment (RESORCE): a randomised, double-blind, placebo-controlled, phase 3 trial. Lancet. 2017;389(10064):56-66. doi:10.1016/S0140-6736(16) 32453-9

26. Abou-Alfa GK, Meyer T, Cheng AL, et al. Cabozantinib in patients with advanced and progressing hepatocellular carcinoma. $N$ Engl J Med. 2018;379(1):54-63. doi:10.1056/NEJMoa1717002

27. Sanchez-Salcedo P, De-torres JP, Martinez-Urbistondo D, et al. The neutrophil to lymphocyte and platelet to lymphocyte ratios as biomarkers for lung cancer development. Lung Cancer. 2016;97:28-34. doi:10.1016/j.lungcan.2016.04.010

28. Absenger G, Szkandera J, Pichler M, et al. A derived neutrophil to lymphocyte ratio predicts clinical outcome in stage II and III colon cancer patients. Br J Cancer. 2013;109(2):395-400. doi:10.1038/ bjc. 2013.346

29. Balta S, Unlu M, Arslan Z, Demırkol S. Neutrophil-to-lymphocyte ratio in prognosis of gastric cancer. $J$ Gastric Cancer. 2013;13 (3):196-197. doi:10.5230/jgc.2013.13.3.196

30. Dirican A, Kucukzeybek BB, Alacacioglu A, et al. Do the derived neutrophil to lymphocyte ratio and the neutrophil to lymphocyte ratio predict prognosis in breast cancer? Int J Clin Oncol. 2015;20 (1):70-81. doi:10.1007/s10147-014-0672-8

31. Feng JF, Huang Y, Liu JS. Combination of neutrophil lymphocyte ratio and platelet lymphocyte ratio is a useful predictor of postoperative survival in patients with esophageal squamous cell carcinoma. Onco Targets Ther. 2013;6:1605-1612.

32. $\mathrm{Hu} \mathrm{H}$, Yao X, Xie X, et al. Prognostic value of preoperative NLR, dNLR, PLR and CRP in surgical renal cell carcinoma patients. World J Urol. 2017;35(2):261-270. doi:10.1007/s00345-016-1864-9

33. McNamara MG, Templeton AJ, Maganti M, et al. Neutrophil/lymphocyte ratio as a prognostic factor in biliary tract cancer. Eur $J$ Cancer. 2014;50(9):1581-1589. doi:10.1016/j.ejca.2014.02.015

34. Lu SD, Wang YY, Peng NF, et al. Preoperative ratio of neutrophils to lymphocytes predicts postresection survival in selected patients with early or intermediate stage hepatocellular carcinoma. Medicine. 2016;95(5):e2722. doi:10.1097/MD.0000000000002722

35. Bishayee A. The role of inflammation and liver cancer. Adv Exp Med Biol. 2014;816:401-435.

36. Liang W, Ferrara N. The complex role of neutrophils in tumor angiogenesis and metastasis. Cancer Immunol Res. 2016;4 (2):83-91. doi:10.1158/2326-6066.CIR-15-0313

37. Ménétrier-Caux C, Ray-Coquard I, Blay JY, Caux C. Lymphopenia in cancer patients and its effects on response to immunotherapy: an opportunity for combination with cytokines? J Immunother Cancer. 2019;7(1):85.

38. Shigeta K, Datta M, Hato T, et al. Dual programmed death receptor-1 and vascular endothelial growth factor receptor-2 blockade promotes vascular normalization and enhances antitumor immune responses in hepatocellular carcinoma. Hepatology. 2020;71(4):1247-1261. doi:10.1002/hep.30889

39. Socinski MA, Jotte RM, Cappuzzo F, et al. Atezolizumab for first-line treatment of metastatic nonsquamous NSCLC. $N$ Engl $J$ Med. 2018;378(24):2288-2301. doi:10.1056/NEJMoa1716948 


\section{Publish your work in this journal}

Cancer Management and Research is an international, peer-reviewed open access journal focusing on cancer research and the optimal use of preventative and integrated treatment interventions to achieve improved outcomes, enhanced survival and quality of life for the cancer patient.

The manuscript management system is completely online and includes a very quick and fair peer-review system, which is all easy to use. Visit http://www.dovepress.com/testimonials.php to read real quotes from published authors. 